\title{
Comparison of Bit Error Performance of Rotated PSK Scheme in Rayleigh and Ricean Fading Channels
}

\author{
Kapil Gupta and P. K. Ghosh
}

\begin{abstract}
In this paper, we study the modulation diversity in PSK scheme obtained by rotating the signal constellation and incorporating bit interleaving. We derive here the expressions for the average bit error rate (ABER) of rotated PSK modulation over frequency non-selective slowly fading Rayleigh and Ricean channels using moment generating function (MGF) based approach. Numerical results are obtained to compare the performance of conventional and rotated modulation schemes. We investigate the dependence of bit error rate on signal-to-noise ratio, fading parameters and the angle of rotation. The results show the improved performance of rotated scheme over conventional method in both the fading models. Also significant gain is noted for the case of strong LOS component. We also find and discuss the optimum rotation angle in rotated PSK scheme. The symmetrical nature of BER with rotation angle is observed.
\end{abstract}

Index Terms-Average bit error rate, bit interleaving, diversity, moment generating function, rayleigh fading channel, rotating signal constellation.

\section{INTRODUCTION}

Fading is an important issue in wireless communication channels. This causes significant degradation of the performance of wireless digital communication systems. High rate date transmission needs to combat fading effects in the channel. One way to reduce the effect of fading is to apply diversity techniques on the communication systems [1]-[5]. Diversity can be implemented as space, time, frequency, code and modulation [6]-[8]. Diversity refers to two or more signal paths that fade signals independently. These independent paths are combined in such a way that the fading of resultant signal is reduced [9], [10]. There are many combining techniques which can apply at the receiver, results in higher SNR per bit. For PSK transmission through wireless medium fading is an important factor for signal degradation and error performance evaluation.

In recent years, modulation diversity obtained by applying a certain rotation to the signal constellation, has attracted great attention to the researchers [11], [12]. Rotation diversity makes use of an interleaver/de-interleaver pair with in-phase and quadrature components of the received signals being affected by independent channel fading coefficients. These fading coefficients called channel state information (CSI) are assumed known at the receiver. The pair-wise error probability based on Chernoff bounds was studied by many authors [6], [12], [13]. When the received signal is composed of signals from different reflected paths in addition to a

Manuscript received September 30, 2011; revised December 12, 2012.

Both the authors are with the Mody Institute of Technology and Science (Deemed University), Lakshmangarh, Dist. Sikar, Rajasthan 332311, INDIA (e-mail: kapil_mbm@yahoo.com; pkghosh_ece@yahoo.co.in ). significant LOS (no-fading) component, the signal envelope has been proved to follow Ricean pdf for small scale fading effect [9], [14].

Of the coherent scheme, PSK modulation is very often preferred due to its better spectral efficiency. In this paper, the closed form expressions for error probabilities for rotated PSK on frequency non-selective Rayleigh and Ricean channel have been derived. Pair-wise error probability is also derived on the moment generating function (MGF) approach for both types of channel fading. The rest of the paper is organized as follows: Section II describes the model of the system using binary PSK over Rayleigh and Ricean fading channels. The Error performance analysis of rotated PSK modulation is presented and discussed in section III. Computed results are presented and discussed in Section IV. The paper finally ends in section $\mathrm{V}$ with some concluding remarks.

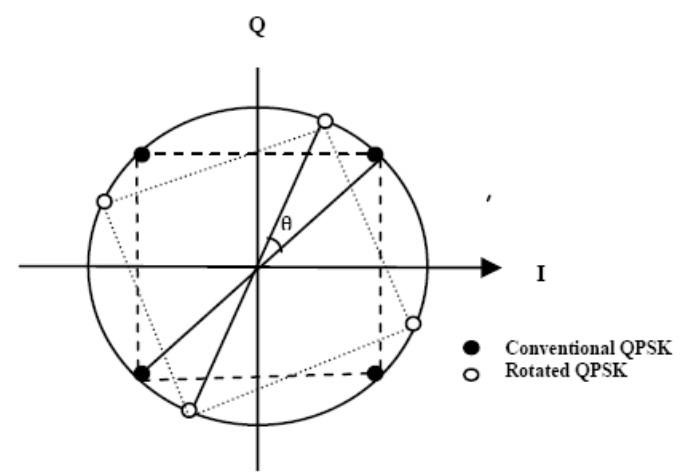

Fig. 1. Signal constellation for conventional QPSK (unrotated) and rotated QPSK.

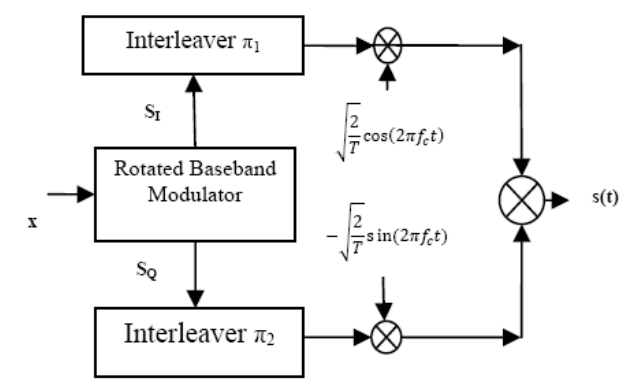

Fig. 2. Block diagram of rotated PSK transmitter.

\section{PERFORMANCE ANALYSIS}

A certain rotation by a constant phase angle to the conventional PSK signal constellation results in rotated PSK signal constellation, as shown in figure1. The small solid circles in Fig. 1 represents the signal points for conventional PSK modulation whereas, the open circles show the case when the signal constellation is rotated by a $\theta^{0}$ angle. The 
conventional PSK has always one common component with some other symbols in the constellation and so symbol interleaving does not offer appreciable SNR gain. But when rotation is applied, the symbols do not have common components and so symbol interleaving introduces a diversity gain into a system [15].

As a result of rotation of signal constellation and symbol interleaving, rotated modulation scheme should have a different transmitter structure as shown in fig. 2. The rotated PSK modulator generates the orthogonal I and Q channels, as shown. The in-phase $\left(\mathrm{s}_{\mathrm{I}}\right)$ and quadrature $\left(\mathrm{s}_{\mathrm{Q}}\right)$ components of the transmitted signal on two channels are independent from each other. The transmitted signal thus can be written as

$$
\begin{aligned}
\mathrm{s}(\mathrm{t})= & \sum_{n=-\infty}^{+\infty} a_{n} p(t-n T) \cos \left(2 \pi f_{c} t\right)+ \\
& \sum_{-\infty}^{+\infty} b_{n} p(t-n T) \sin \left(2 \pi f_{c} t\right)
\end{aligned}
$$

with $a_{n}, b_{n}= \pm 1 \quad$ with equal probability and $p(t)=$ $\left\{\begin{array}{l}1,0 \leq t \leq T \\ 0, \text { elsewhere }\end{array}\right.$ representing the shape of the pulse. In the above equation, $f_{c}$ is the carrier frequency and $T$ is the symbol duration. Because of rotating signal constellation, the bit-interleaved transmitted signal can be written as

$$
\begin{array}{r}
\mathrm{s}(\mathrm{t})=A \sum_{n=-\infty}^{+\infty} x_{n} p(t-n T) \cos \left(2 \pi f_{c} t\right)+ \\
A \sum_{-\infty}^{+\infty} y_{n} p(t-n T) \sin \left(2 \pi f_{c} t\right)
\end{array}
$$

where $x_{n}=a_{n} \cos \theta-b_{n} \sin \theta$, and $y_{n}=a_{n} \sin \theta+$ $b_{n} \cos \theta$ and $A$ represents the modulator gain. The above modulated signal is now transmitted through the transmission medium which is assumed to be additive white Gaussian noise (AWGN) channel. The interleavers are selected such that both the in-phase and quadrature components are independent after de-interleaving. The rotating phase angle is chosen such that the squared Euclidian distance between signals constellations are maximized for both the components.

The closest constellation points for BPSK scheme depends on the rotation angle. Here we take a digital communication channel to be frequency non-selective, slowly fading with multiplicative fading envelope and AWGN component. The received signal is the faded and noisy version of the transmitted signal $s(t)$ and is can be written as

$$
r(t)=\alpha(t) s(t)+n(t)
$$

where $\alpha(t)$ represents fading envelope causing random amplitude variation and $\mathrm{n}(\mathrm{t})$ represents zero mean Gaussian noise. In case of slow fading, the fading amplitude is regarded constant over one symbol interval. The block diagram for the receiver structure of rotated PSK scheme is sketched in figure 3 . There are a number of statistical models available in the literature to characterize fading envelopes under different channel conditions [16].

We study here the Rayleigh and Ricean distributed fading components due to their wide applications in wireless environments. If the received signal contains strong line-of-sight (LOS) component, the fading distribution is taken to be Ricean [16]. The effect of the fading is decided by the Ricean factor which is defined as the ratio of LOS to the non- LOS components. The received signal is first applied to the correlated demodulators (two orthogonal carriers $\cos \left(2 \pi f_{c} t\right)$ and $\sin \left(2 \pi f_{c} t\right)$ multiply the received signal in two different branches) and then de-interleaved. The outputs of de-inteleavers are $r_{I} \& r_{Q}$, the in-phase and quadrature components of the received vector $\bar{r}$ given by

$$
\bar{r}=\left(r_{I}, r_{Q}\right)=\left(\alpha_{1} s_{I}+n_{l}, \alpha_{2} s_{Q}+n_{Q}\right)
$$

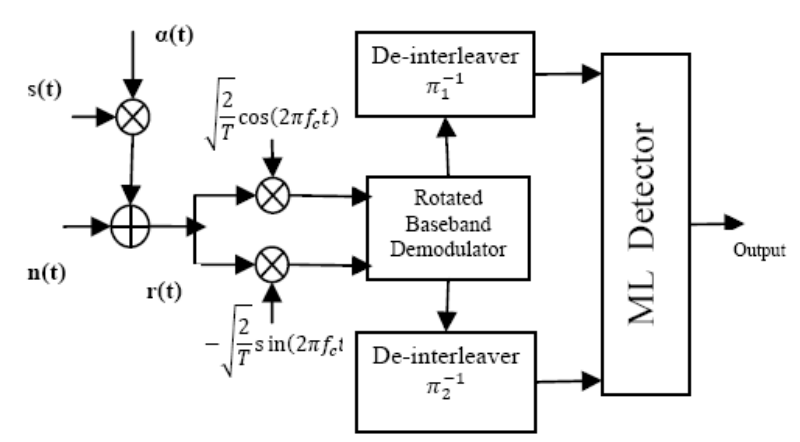

Fig. 3. Block diagram of PSK receiver.

In case of ideal interleavers with $\alpha_{1}$ and $\alpha_{2}$ as independent identically distributed (i.i.d) random variables of zero mean with $E\left[\alpha_{1}^{2}\right]=E\left[\alpha_{2}^{2}\right]=1$. Note that the means of $\alpha_{1}$ and $\alpha_{2}$ are non-zero for Ricean distributed random variables. The quantities $n_{I}$ and $n_{Q}$ representing in-phase and quadrature noise components, are assumed independent random variables with zero mean and variance $\mathrm{N}_{0} / 2$. The complete channel state information (CSI) is assumed available at the receiver and the detection is based on maximum likelihood ML decision [17]. The corresponding detection metric $m\left(\overline{s_{l}} \mid \bar{r}, \bar{\alpha}\right)$ can be obtained as

$$
m\left(\overline{s_{l}} \mid \bar{r}, \bar{\alpha}\right)=\left\|\bar{r}-\bar{\alpha} \odot \overline{s_{l}}\right\|^{2}, i=1,2
$$

where $\bar{\alpha}=\left(\alpha_{1}, \alpha_{2}\right)$ and $\mathrm{s}_{\mathrm{i}}(\mathrm{i}=1,2)$ is the $\mathrm{i}^{\text {th }}$ symbol of rotated BPSK constellation, ' $\|$. $\|$ ' denotes the norm and $\odot$ represents component-wise product.

\section{PERFORMANCE ANALYSIS}

The conditional bit error probability can be written as

$$
P\left(\bar{s} \rightarrow \hat{s} \mid \alpha_{1}, \alpha_{2}\right)=Q\left(\sqrt{\frac{E_{s} \alpha_{1}^{2} \Delta_{I}^{2}+E_{S} \alpha_{2}^{2} \Delta_{Q}^{2}}{2 N_{0}}}\right)
$$

where the vector $\hat{\mathrm{s}}=\left(\hat{\mathrm{s}}_{\mathrm{I}}, \hat{\mathrm{s}}_{\mathrm{Q}}\right)$ denote the in-phase and quadrature components of the estimate of the signal $\mathrm{s}$, $\Delta_{I}=s_{I}-\hat{s}_{I}, \Delta_{Q}=s_{Q}-\hat{s}_{Q}$, and $\mathrm{Q}($.$) is the Gaussian Q-$ function defined by

$$
\mathrm{Q}(\mathrm{x})=\frac{1}{\sqrt{2 \pi}} \int_{\mathrm{x}}^{\infty} \mathrm{e}^{-\mathrm{t}^{2} / 2} \mathrm{dt}
$$

The above conditional probability of error event can be averaged out over pdf of fading amplitudes to get average bit error probability. By taking $y_{1}=\frac{\alpha_{1}^{2} \Delta_{I}^{2}}{N_{0}}$ and $y_{2}=\frac{\alpha_{2}^{2} \Delta_{Q}^{2}}{N_{o}}$, the average bit error rate (ABER) can be obtained as [16] 


$$
P(\bar{s} \rightarrow \hat{s})=\int_{0}^{\infty} \frac{1}{\pi} \int_{0}^{\pi / 2} \exp \left(\frac{-y}{4 \sin ^{2} \varnothing}\right) d \emptyset p_{y}(y) d y
$$

where $p_{y}(y)$ is the pdf of $\mathrm{y}=\mathrm{y}_{1}+\mathrm{y}_{2}$ and $\mathrm{M}_{\mathrm{y}}(\beta)$ is the moment generating function of $y$, defined as

$$
M_{y}(\beta)=\int_{0}^{\infty} p_{y}(y) e^{\beta y} d y
$$

So we can write equation (8) as

$$
P(\bar{s} \rightarrow \hat{s})=\frac{1}{\pi} \int_{0}^{\pi / 2} M_{y}\left(\frac{-1}{4 \sin ^{2} \emptyset}\right) d \emptyset
$$

The MGF of Rayleigh distributed random variable is given by [16]

$$
M_{y}(\beta)=(1-\beta \bar{y})^{-1}
$$

The MGF of y is given by [18], which can be expressed as

$$
M_{y}(\beta)=M_{y 1}(\beta) \cdot M_{y 2}(\beta)
$$

\section{A. Error Analysis for Rayleigh fading Channel}

By Eq. (12), the MGF of y is written as

$M_{y}\left(\beta=\frac{-1}{4 \sin ^{2} \emptyset}\right)=\left(\frac{\sin ^{2} \emptyset}{\sin ^{2} \emptyset+\frac{\bar{y}_{1}}{4}}\right)\left(\frac{\sin ^{2} \emptyset}{\sin ^{2} \emptyset+\frac{\overline{y_{2}}}{4}}\right)$

Thus conditional error probability is

$$
P(\bar{s} \rightarrow \hat{s})=\frac{1}{\pi} \int_{0}^{\infty}\left(\frac{\sin ^{2} \emptyset}{\sin ^{2} \emptyset+\frac{\overline{y_{1}}}{4}}\right)\left(\frac{\sin ^{2} \emptyset}{\sin ^{2} \emptyset+\frac{\overline{y_{2}}}{4}}\right) \mathrm{d} \emptyset
$$

By substituting $c_{1}=\frac{\overline{y_{1}}}{4}, c_{2}=\frac{\overline{y_{2}}}{4}$ and $\mathrm{x}=\cos ^{2} \emptyset$, the above equation reduces to

$$
\begin{gathered}
P(\bar{s} \rightarrow \hat{s}) \\
=\frac{1}{2 \pi\left(1+c_{1}\right)\left(1+c_{2}\right)} \int_{0}^{1}(1-x)^{1.5} x^{-.5}(1-u x)^{-1}(1-v x)^{-1} d x
\end{gathered}
$$

where $u=\frac{1}{\left(1+c_{1}\right)}$ and $v=\frac{1}{\left(1+c_{2}\right)}$

The ABER can be expressed in the closed form [19, Eq 3.211]

$$
\begin{gathered}
P(\bar{s} \rightarrow \hat{s}) \\
=\frac{B(2.5,0.5)}{2 \pi\left(1+c_{1}\right)\left(1+c_{2}\right)} F_{1}\left(0.5,1,1,3 ;\left(1+c_{1}\right)^{-1},\left(1+c_{2}\right)^{-1}\right)
\end{gathered}
$$

(16)

where $F_{1}(., \ldots, ., \ldots)$ is Appell hypergeometric function and $\mathrm{B}(\mathrm{a}, \mathrm{b})$ is the beta function.

At high SNR, the hypergeometric fuction converges to one and the upper bound of error rate can be expressed as

$$
\begin{gathered}
P(\bar{s} \rightarrow \hat{s}) \leq \frac{B(2.5, .5)}{2 \pi\left(c_{1}\right)\left(c_{2}\right)} \\
=\frac{B(2.5, .5)}{2 \pi\left(\frac{\alpha_{1}^{2} \Delta_{I}^{2}}{4 N_{0}}\right)\left(\frac{\alpha_{2}^{2} \Delta_{Q}^{2}}{4 N_{o}}\right)}=\frac{B(2.5,5)}{2 \pi\left(\frac{\alpha_{1}^{2} \alpha_{2}^{2}}{N_{0}}\right)\left(\frac{\Delta_{I} \Delta_{Q}}{4}\right)^{2}}
\end{gathered}
$$

For binary symbols $s_{1}$ and $s_{2}$, the above equation simplifies to

$$
P_{s}=\frac{1}{2}\left[P\left(s_{2} \rightarrow s_{1}\right)+P\left(s_{1} \rightarrow s_{2}\right)\right]
$$

\section{B. Error Analysis for Ricean fading Channel}

The MGF of $\mathrm{y}$ is given by [16]

$M_{y}(\beta)=\frac{1+n^{2}}{\left(1+n^{2}\right)-\beta \bar{y}} \exp \left(\frac{n^{2} \beta \bar{y}}{\left(1+n^{2}\right)-\beta \bar{y}}\right)$

Let $K=n^{2}+1=a+1$, where $n^{2}=a$ is the Ricean factor. Then,

$M_{y}(\beta)=\frac{K}{K-\beta \bar{y}} \exp \left(\frac{(K-1) \beta \bar{y}}{K-\beta \bar{y}}\right)$

By substituting $\beta=\frac{-1}{4 \sin ^{2} \emptyset}$ in the above equation, we obtain

$$
\begin{aligned}
M_{y}\left(\beta=\frac{-1}{4 \sin ^{2} \varnothing}\right) & =\frac{K}{K-\left(\frac{-1}{4 \sin ^{2} \varnothing}\right) \bar{y}} \exp \left(\frac{(K-1)\left(\frac{-1}{4 \sin ^{2} \phi}\right) \bar{y}}{K-\left(\frac{-1}{4 \sin ^{2} \varnothing}\right) \bar{y}}\right) \\
& =\frac{\sin ^{2} \varnothing}{\sin ^{2} \emptyset+c} \exp \left(\frac{(1-K) c}{\sin ^{2} \emptyset+c}\right)
\end{aligned}
$$

where $c=\frac{\bar{y}}{4 K}$. By Eq.(12), the MGF of y can be written as

$$
M_{y}(\beta)=\frac{\sin ^{4} \emptyset}{\left(\sin ^{2} \emptyset+c_{1}\right)\left(\sin ^{2} \emptyset+c_{2}\right)} \exp \left[\left(\frac{(1-K) c_{1}}{\sin ^{2} \emptyset+c_{1}}\right)+\left(\frac{(1-K) c_{2}}{\sin ^{2} \emptyset+c_{2}}\right)\right]
$$

The conditional error probability is thus

$$
\begin{gathered}
P(\bar{s} \rightarrow \hat{s})=\frac{1}{\pi} \int_{0}^{\pi / 2} \frac{\sin ^{4} \emptyset}{\left(\sin ^{2} \emptyset+c_{1}\right)\left(\sin ^{2} \emptyset+c_{2}\right)} \exp \left[\left(\frac{(1-K) c_{1}}{\sin ^{2} \emptyset+c_{1}}\right)+\right. \\
\left.\left(\frac{(1-K) c_{2}}{\sin ^{2} \emptyset+c_{2}}\right)\right] d \emptyset
\end{gathered}
$$

By substituting $c_{1}=\frac{\overline{y_{1}}}{4 \mathrm{~K}}, c_{2}=\frac{\overline{y_{2}}}{4 \mathrm{~K}}$ and $\mathrm{x}=\cos ^{2} \emptyset$, the above equation reduces to

$$
\begin{gathered}
P(\bar{s} \rightarrow \hat{s})=\frac{1}{2 \pi} \int_{0}^{1} \frac{(1-x)^{1.5}}{(x)^{.5}\left(1-x+c_{1}\right)\left(1-x+c_{2}\right)} \exp \left[\left(\frac{(1-K) c_{1}}{1-x+c_{1}}\right)+\right. \\
\left.\left(\frac{(1-K) c_{2}}{1-x+c_{2}}\right)\right] d x
\end{gathered}
$$

This is the expression we get for ABER when fading is Ricean distributed.

The bit error probability being a function of rotation angle $\theta$, the ABER is required to be minimized with respect to $\theta$ for achieving better performance. When $\theta=0^{0}$ (without rotation), the PSK signal constellations are pair-wise symmetric with respect to the two axes and the performance of the system reduces to conventional BPSK.

\section{NumericAl RESUlts}

Fig. 4 depicts the bit error rate versus SNR (dB) in Rayleigh fading channel for different values of rotation angle $\theta^{\circ}$. The upper curve is for conventional BPSK (un-rotated) scheme. As the rotation angle increases from $0^{\circ}$ to $45^{\circ}$ the system performance improves that is, the BER decreases with the increase in SNR values. At the BER of $10^{-3}$, the SNR gain improves by $7.0 \mathrm{~dB}$ for $15^{\circ}, 10 \mathrm{~dB}$ for $30^{\circ}$ and $10.3 \mathrm{~dB}$ for $45^{\circ}$. A further increase in the rotation angle beyond $45^{\circ}$ lowers the performance index due to the symmetrical dependency of BER on rotation angle. When the angle of rotation is increased beyond $45^{\circ}$, the values of $u$ and $v$ in Eq. (13) are interchanged. This gives identical values of $P(\bar{s} \rightarrow \hat{s})$ for 
complementary angles. The $(*)$ marks on the curves for $15^{0}$ and $30^{\circ}$ indicate the values obtained for $75^{\circ}$ and $60^{\circ}$, respectively. The figure also reflects that at a typical SNR of $24 \mathrm{~dB}$, the BER is $9.5^{*} 10^{-4}$ for $\theta=0^{\circ} ; 4.2^{*} 10^{-5}$ for $\theta=15^{\circ} \&$ $75^{\circ} ; 1.47^{*} 10^{-5}$ for $\theta=30^{\circ} \& 60^{\circ}$; and $1.11^{*} 10^{-5}$ for $\theta=45^{\circ}$.

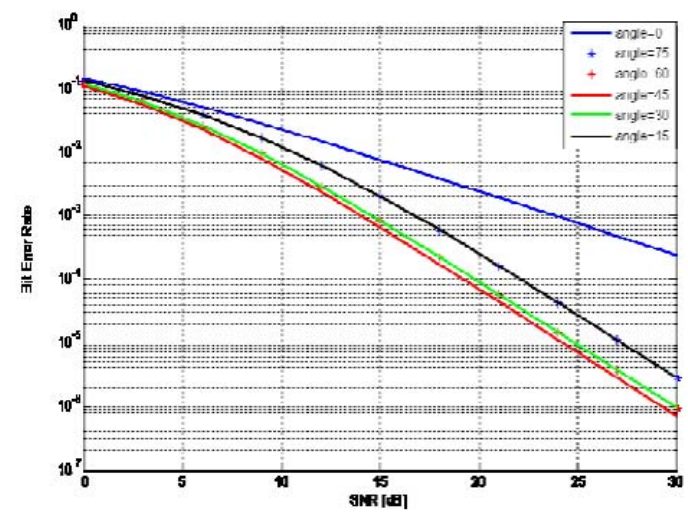

Fig 4. Plot of average BER with SNR (dB) for rayleigh fading for both conventional and rotated BPSK for different angles of rotation.

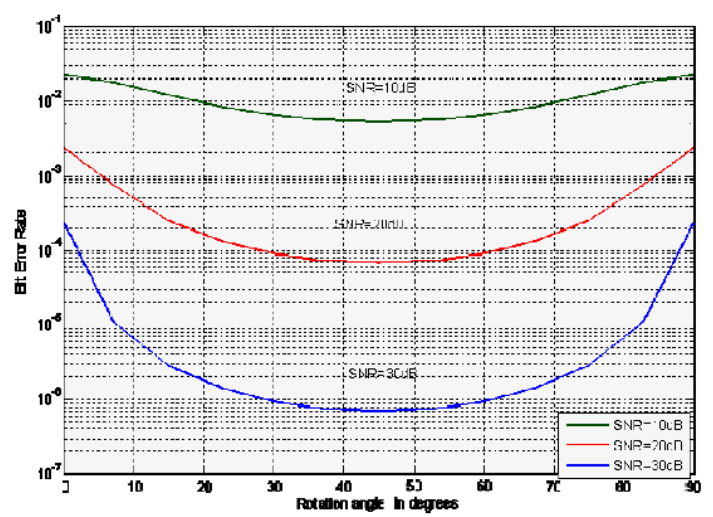

Fig. 5. Variation of BER with rotation angle $\theta$ for fixed SNR (dB) in case of rayleigh fading channel.

Fig 5 shows the variation of BER with rotation angle $\theta$ for SNR values of 10,20 and $30 \mathrm{~dB}$. The minimum value of BER is observed at $\theta=45^{\circ}$ for all the values of SNR. As the SNR increases, the BER decreases as expected. The study of BER performance for Ricean channel has also been undertaken; the comparison will be made in Fig. 8.

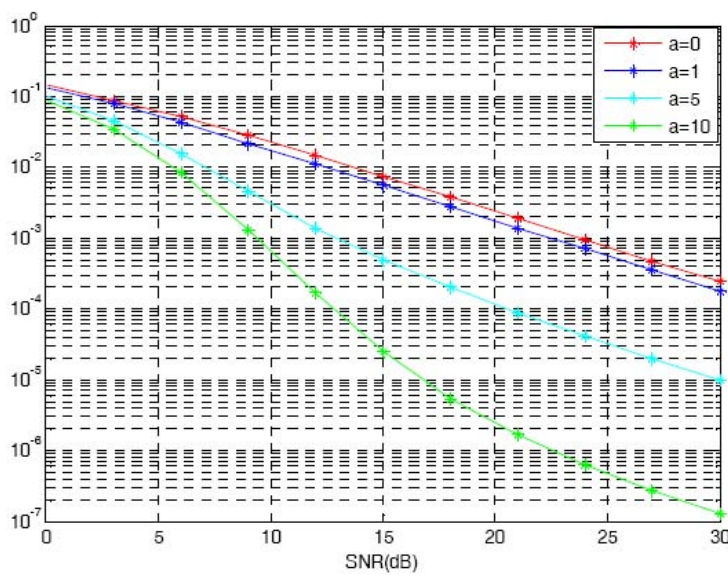

Fig 6. Change of bit error rate on $\operatorname{SNR}(\mathrm{dB})$ for un-rotated BPSK $\left(\theta=0^{\circ}\right)$ for different values of ricean factor.

The BER performance of un-rotated BPSK scheme for different values of Ricean factor $(a=0,1,5$, and 10) is depicted in Fig. 6. For a comparison, the plot for $\mathrm{a}=0$ corresponds to the Rayleigh fading as shown in Fig. 4. At a given SNR value, the BER decreases as Ricean factor increases due to strong LOS component. At a typical BER of $10^{-3}$, the Ricean BPSK is $1.5 \mathrm{~dB}, 11 \mathrm{~dB}$, and $15 \mathrm{~dB}$ superior to Rayleigh fade-limited scheme for Ricean factors of $a=1,5$, and10 respectively. Further, at a fixed SNR of $25 \mathrm{~dB}$, the BER for the Ricean factors of $a=0,1,5$, and 10 are $7 * 10^{-4}$, $5 * 10^{-4}, 3 * 10^{-5}$ and $5 * 10^{-7}$ respectively.

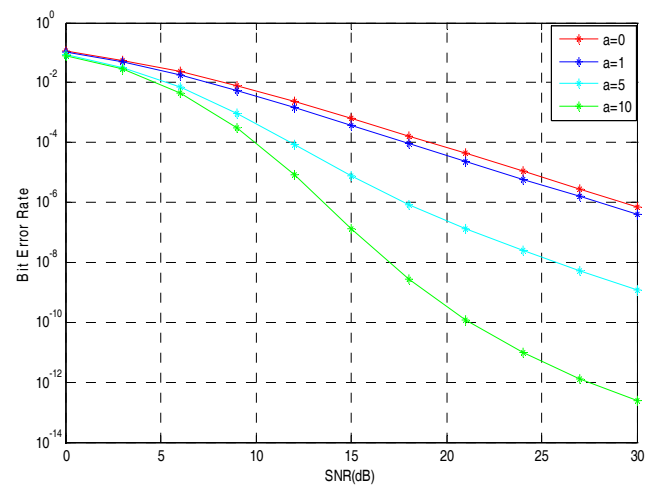

Fig. 7. Bit error rate versus SNR $(\mathrm{dB})$ for un-rotated BPSK and rotated BPSK for different values of Ricean factor at an angle of $\theta=45^{\circ}$.

BER results for non-zero rotation angle are shown in Fig. 7 for $\theta=45^{\circ}$ where the bit error rate is lower than any other $\theta$-values. Compared to the Rayleigh-faded BPSK with $\theta=0^{0}$ and for the BER of $10^{-3}$, the Ricean-faded BPSK SNR improves by $1.5 \mathrm{~dB}, 5 \mathrm{~dB}$, and $6.5 \mathrm{~dB}$ for $a=1,5$, and 10 , respectively. We also observe that at a fixed SNR, the error rate improves for higher Ricean factors.

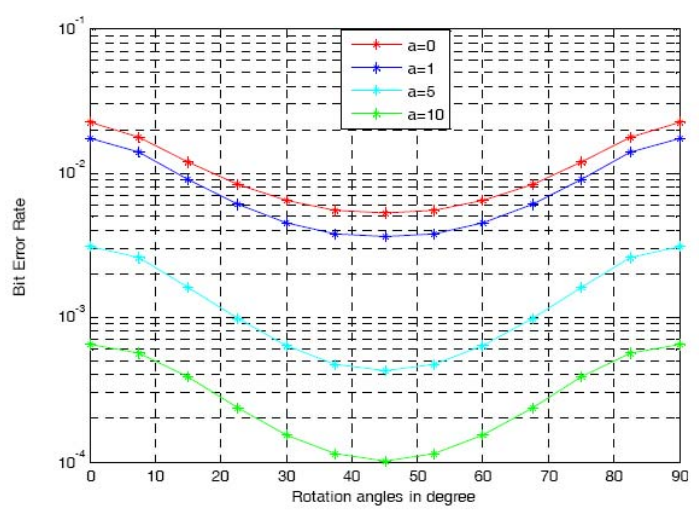

Fig. 8. Variation of BER with rotation angle $\theta$ for fixed $S N R=10 \mathrm{~dB}$.

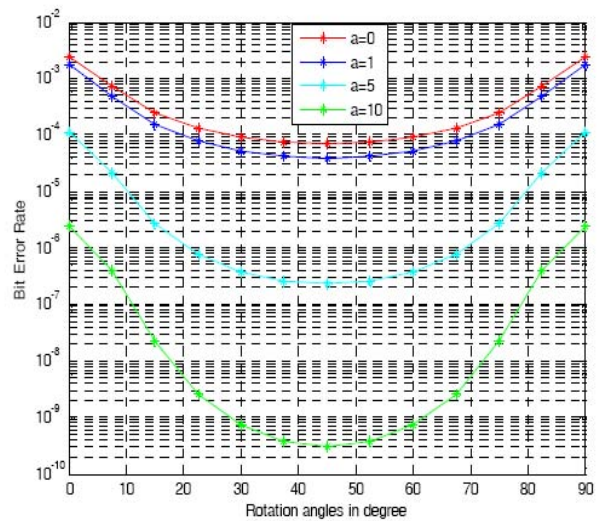

Fig 9. Variation of BER with rotation angle $\theta$ for fixed $\mathrm{SNR}=20 \mathrm{~dB}$. 
Fig 8 shows the variation of BER with rotation angle $\theta$ for a fixed SNR of $10 \mathrm{~dB}$ and with different values of Ricean factor $(a=0,1,5$, and 10). In this case the minimum value of BER is observed at $\theta=45^{\circ}$ for all the values of Ricean factor. Similar calculations at an SNR of $20 \mathrm{~dB}$ as depicted in Fig. 9 show the improved error performance.

\section{CONCLUSION}

In this paper, we have derived the expressions for bit error analysis using moment generating function for conventional and rotated PSK modulation schemes over Rayleigh and Ricean faded channels. We have studied the dependence of bit error rates on the modulation techniques, signal-to-noise ratio, fading parameters and the angle of rotation. As the SNR increases, the BER decreases as expected. Our critical analysis shows that the bit error rate attains a minimum for the rotation angle of $45^{\circ}$ and improves at higher SNR for both the fading models. The symmetrical variation of BER versus rotation angle about $45^{\circ}$ is observed and explained. It is also found that significant improvement in error probability occurs when the signal is enriched with strong LOS component.

\section{ACKNOWLEDGEMENT}

The authors wish to thank Anup Dey, Department of Electronics and Communication Engineering of Kalyani Government Engineering College, West Bengal, India for his valuable suggestions and discussions.

\section{REFERENCES}

[1] T. A. Tsiftsis, "Performance of Wireless Multihop Communications Systems with Cooperative Diversity over Fading Channels", International Journal of Communications Systems, Wiley, vol. 20, no.12, Dec. 2007.

[2] J. N. Laneman, D. N. C. Tse, and G. W. Wornell, "cooperative diversity in wireless networks: efficient protocols and outage behavior," IEEE Trans. Inform. Theory, Vol. 50, no.12, pp. 3062-3080, Dec. 2004.

[3] D. Chen and J. N. Laneman, "Modulation and demodulation for cooperative diversity in wireless systems," IEEE Trans. Wireless Commun., vol.5, no.5, pp. 1785-1794, July 2006.

[4] Bletsas, A., Khisti, A., Reed, D.P., and Lippman, A. "A simple cooperative diversity method based on network path selection," IEEEJournal on SelectedAareas in Commun., vol. 24, no. 3, March 2006.

[5] Z. Ying, T. Bolin, V. Plicanic, A. Derneryd, and G. Kristensson," Diversity antenna terminal evaluation," in proc. of IEEE Antennas Propagation Society International Symposium, Washington D.C., 2A:375-378, 2005.

[6] Y. Jing and H. Jafarkhani, "Single and multiple relay selection schemes and their achievable diversity orders", IEEE Trans. on Wireless Communications, Vol. 8, no. 3, pp. 1414-1423, 2009.

[7] Q. Zhou and H. Dai, "Asymptotic analysis on the interaction between spatial diversity and multiuser diversity in wireless networks," IEEE Trans. on Signal Processing, Vol. 55, no. 8, pp. 4271-4283, 2007.
[8] M. Uysal, "Diversity analysis of space-time coding in Cascaded Rayleigh Fading Channels," IEEE Commumn. Let. vol.10, no. 3, pp. 165-167, March 2006.

[9] Andrea Goldsmith, Wireless Communications, Cambridge University Press, 2005.

[10] S. Pornchai, W. Wannaree, and T. Sawasd, "Performance of M-PSK in Mobile Satellite Commuincation over Combined Ionospheric Scintillation and Flat Fading Channels with MRC Diversity," IEEE Transactions on Wireless Communications. Vol. 8(7):3360-3364, 2009.

[11] S. $\ddot{Q}$ zyurt, O. Kuchur, $\dot{I}$. brahim Altunbas, "Error performance of rotated phase shift keying modulation over fading channels," Wireless Pers. Commun. vol. 43, pp. 1453-1463, 2007.

[12] N. F Kiyani, U. H. R izvi, G. Dolmans, "Modulation diversity benefits in cooperative communications", Wireless Telecommunications Symposium (WTS), pp: 1-5, 21-23 April 2010.

[13] S. B. S limane, "An impoved PSK scheme for fading channels", IEEE Transaction on Vehicular Technology, vol. 47(2), pp-703-710, 1998.

[14] S. T. Rappaport, Wireless Communications, $2^{\text {nd }}$ edition, Peason, 2009.

[15] A. Yilmaz and O. Kucur, "Performance analysis of rotated PSK over Nakagami-m fading channels", IEEE 6 th Int. Symp. on Wireless Commun. Systems, Siena, Italy, September pp. 7-10, 2009.

[16] M. K. Simon and M. S. Alouini, Digital Communication over Fading Channels, 2nd edition, Wiley and Sons, Inc, 2005

[17] G. John Proakis, Digital Communications, McGraw- Hill, New York, 2001.

[18] A. Papoulis, Probability, Random Variables, and Stochastic Processes, $3^{\text {rd }}$ edition, McGraw-Hill, New York, 1991.

[19] I. S. Gradshteyn, I. M. Ryzhik, Table of Integrals, Series, and Products, 5th edition, Academic Press, San Diego, CA,1994.

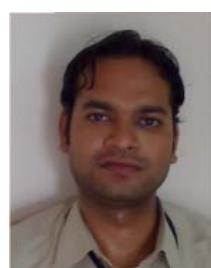

Kapil Gupta was born in Kota (Rajasthan), India in 1980. He received his B.Tech (Hons) degree in Electronics \& Communication Engineering in 2003 from Rajasthan University and M.Tech (Hons) degree in Digital Communication from JNV University, Jodhpur, Rajasthan in 2008. He is an Assistant Professor of Mody Institute of Technology and Science (Deemed University), Lakshmangarh and has 6 years of experience in teaching. His research interests are in Digital Communication over fading channel and Error correction codes

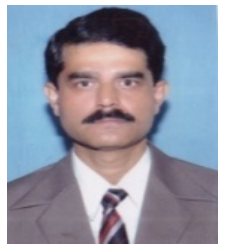

P. K. Ghosh was born in Kolkata, India in 1964 He received his B.Sc (Hons in Physics), B.Tech and M.Tech degrees in 1986, 1989, and 1991, respectively, from Calcutta University. He earned Ph.D.(Tech) degree in Radio Physics and Electronics in 1997 from the same University. He served various institutions, namely, National Institute of Science and Technology (Orissa), St. Xavier's College (Kolkata), Murshidabad College of Engineering and Technology (West Bengal), R. D. Engineering College (Uttar Pradesh) and Kalyani Government Engineering College (West Bengal) before he joins Mody Institute of Technology and Science (Rajasthan). To his credit, he has more than 30 research papers in Journals of repute and conference proceedings. He is life member of Indian Society for Technical Education (ISTE), New Delhi. His research interests are in the areas of wireless communications, reduced order modeling, VLSI circuits and devices. 